\title{
Drainage Vehicle Telescopic pipe joint's Seal Performance Analysis For Inflatable Sealing Ring
}

\author{
Tang Youming ${ }^{1,2}$, LiuNa ${ }^{1}$, Lin Zhiguo ${ }^{3}$, Wu Jinghui ${ }^{3}$
}

(1. College of Mechanical and Automotive Engineering, Xiamen University of Technology, Xiamen City, Fujian Province, P.R.China 361024; 2. Collaborative Innovation Center for R\&D of Coach and Special Vehicle, Xiamen University of Technology, Xiamen City, Fujian Province, P.R.China 361024; 3. Fujian Qiao Long Special Purpose Vehicle Co.Ltd.,Longyan, Fujian Province, P.R.China 364099)

Keywords:T-shaped ring. Drainage.Finite element method.Assembly clearance Abstract : According to the telescoping tube failure problems, and the drainage vehicle displacement is $3000 \mathrm{~m} 3 / \mathrm{h}$, T-shaped inflatable sealing ring parameters were analyzed. Using the finite element software ANSYS to build numerical simulation models. Sealing performance under different inflatable pressures, different assembly spaces and different initial compression ratios is analyzed. The maximum stress, maximum contact stress and maximum shear stress of the sealing ring are the failure criteria. The results showed that different inflation pressure, different assembly clearance and different initial compression ratio have a great influence on the maximum contact stress of the sealing ring. The maximum stress increased with the increasing of inflation pressure and the peak zone of the stress changed when keeping the compression ratio and assembly clearance as a constant. The optimal inflation pressure of the telescoping tube is $0.4 \sim 0.6 \mathrm{mpa}$.

\section{Introduction}

The sealing design and analysis of the telescoping tube, end face, and the circumference of the drainage pipe is attached more and more importance. At present, many scholars have carried out the numerical simulation of the rubber sealing ring by using nonlinear finite element method, but the research on the structure of the pneumatic seal is still relatively small. Foreign Montgomery[1] researched on the sealing and failure mode of the choke pipe line of the water pipe joint. Cary[2] proposed the Sealing failure repair program of the riser joint.AI-Kharusi[3] used ABAQUS to analyze the stress of the elastic body under different pressure in the oil well production. Domestic Xiang Kai Meng[4] used MSC.M arc to analyze the shaped of "a" inflatable sealing structure, Ben Kecun [5] used ANSYS to analyze the "V" shape inflatable flexible sealing ring. But it is very little that specifically for the drainage vehicle telescoping tube of the inflatable sealing ring. In this paper, the ANSYS software is used to finite element analysis for the cross-sectional structure of "T" shaped inflatable flexible ring.

\section{Finite Element Model of Inflation Sealing Ring}

Geometric Model. Fujian Qiao Long "dragon-absorbent" drainage vehicle telescoping tube uses hollow inflatable sealing ring, Cross section is "T" type. The gas in the air reservoir that via a switch, gas pipelines and air nozzle is delivered to the inflatable ring and make the sealing ring toward the telescopic tube side jacked up, so that closes contact with the outer surface of the inner telescopic tube, blocking the sealing gap, so as to achieve the purpose of sealing and to be leak proof, the installation position and pattern of $\mathrm{T}$ - ring in the drainage vehicle as shown in Fig.1. 


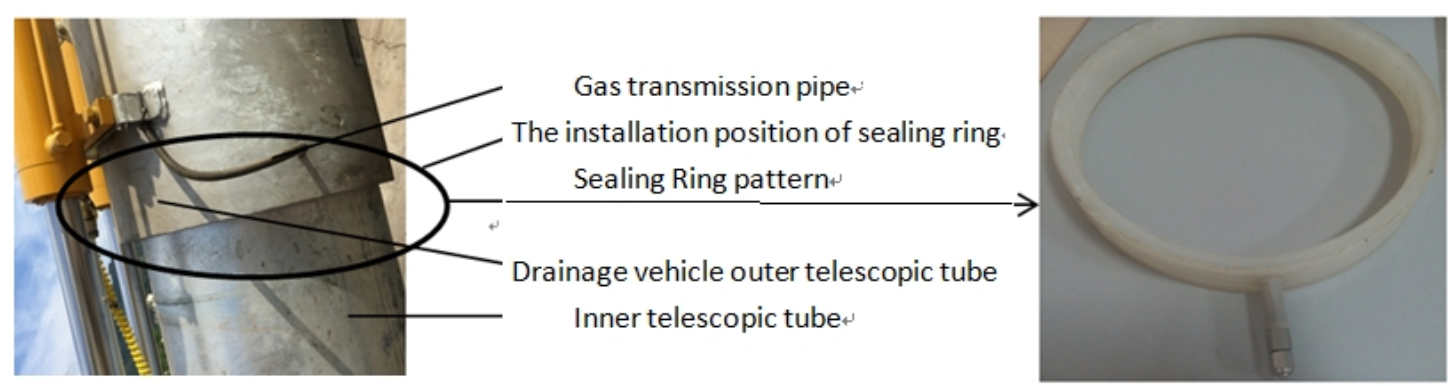

Fig.1 The Installation position and specific pattern of T - ring in the drainage vehicle

Finite element model of inflatable sealing ring is analyzed. The sealing mechanism is divided into three entities, respectively, the card slot, the sealing ring, the interior of telescopic tube. According to the geometry of inflatable sealing ring and material, boundary conditions, building the three dimensional axisymmetric model of rubber, as shown in Fig. 2 (a). Loading pressure on the four inner surfaces of the seal ring makes that full of groove. Putting uniform medium pressure on the inside sealing ring, as shown in Fig. 2 (b).

The constitutive model of rubber material assumptions are as follows: (1) isotropic material, and the deformation is completely restored; (2) material can hardly be compressed. The material of the inflation seal ring is super elastic body and the hardness is HA40, which belongs to the nonlinear material. The Mooney-Rivlin model is used to describe the physical nonlinearity of rubber [7].

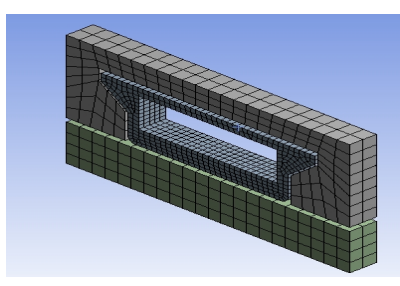

(a) finite element model

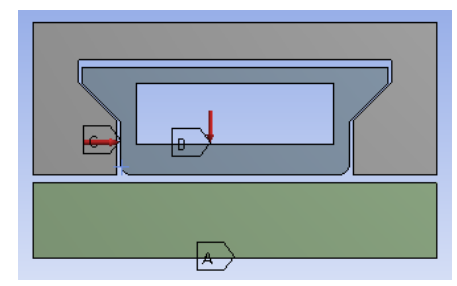

(b) The position of loading force and fixed plane

Fig.2 The finite element model of the sealing ring

\section{Digital Simulation Analysis}

Deformation And Stress Distribution of Sealing Ring. Difference inflation pressures had different effects. The equivalent stress at $0.08,0.1,0.2,0.4,0.6,0.8 \mathrm{mpa}$ is analyzed when the initial expansion ratio is $13 \%$, assembling clearance is $1 \mathrm{~mm}$. The stress distribution of the pneumatic sealing ring under different inflation pressures as shown in Fig.3.

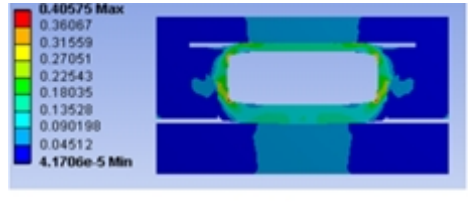

0.08

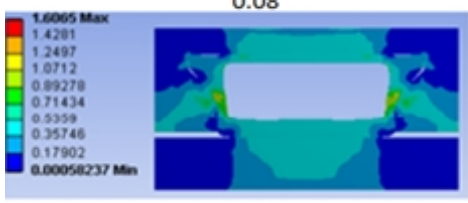

0.4

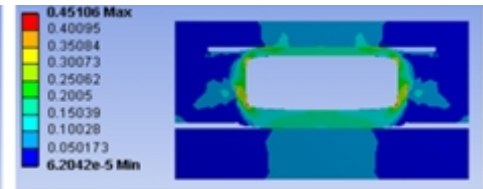

0.1 .
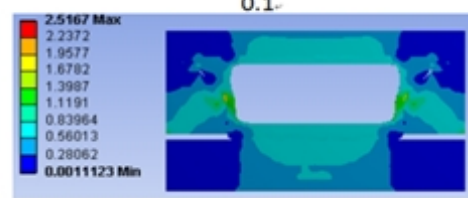

0.6

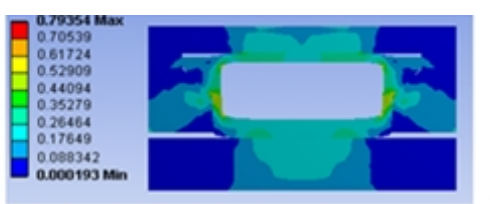

0.2
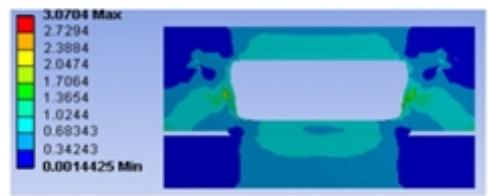

0.8.

Fig.3 Equivalent stress distribution

By graph, the maximum stress equals to $3.0704 \mathrm{mpa}$ under $0.8 \mathrm{mpa}$, which is less than the rubber yield stress $9.240 \mathrm{mpa}$, meets the strength requirements. Contacting length between internal and external drain pipe increases with the pressure's increasing when the compression ratio and 
assembly clearance is constant. The peaking zone of the stress transferred from inside corners to the sharp rigid parts. This shows that the failure position under different inflation pressures will change.

\section{Contact Stress Analysis}

Contact Stress Under Different Inflation Pressures. When the assembling clearance is $1 \mathrm{~mm}$, the initial compression ratio is $13 \%$, and the inflation pressures equal to $0.08,0.2,0.4,0.6$ and $0.8 \mathrm{MPa}$, contact stress's curve as shown in Fig. 4. The inflation pressure is greater than the maximum contact stress at 0.08 or $0.8 \mathrm{MPa}$. Sealing ring can be judged as disable. Too large inflation pressures would make sealing ring happen to warp. The $0.08 \mathrm{mpa}$ don' $\mathrm{t}$ enough to make the sealing ring and end surface tightly contact. The maximum contact stress is always greater than the inflation pressure at $0.1 \sim 0.6 \mathrm{MPa}$. The contact stress and the inner equivalent stress increased when inflation pressure increased. The stress too large will lead to rubber aging, which will affect the service life of the sealing ring. In engineering, the inflation pressure is only higher than $0.15 \sim 0.2 \mathrm{mpa}$ usually [8].

Contact Stress Under Different Assembly Clearance. The assembly clearance is the gap between the inner and outer telescopic tube. The initial compression ratio is $13 \%$ and the clearance is $3 \mathrm{~mm}$, $2 \mathrm{~mm}$ and $1 \mathrm{~mm}$. The contact stress curve in different assembly gaps as shown in Fig. 5. The results show that under the same condition, the smaller the assembly clearance is, the smaller the contact stress when the inflation under $0.25 \mathrm{MPa}$. Above $0.42 \mathrm{mpa}$, the greater the assembly clearance is, the smaller contact stress is. By calculation, when the displacement is $3000 \mathrm{~m}^{3} / \mathrm{h}$, medium pressure is about $0.22 \mathrm{mpa}$, the assembly clearance should be reduced as far as possible.

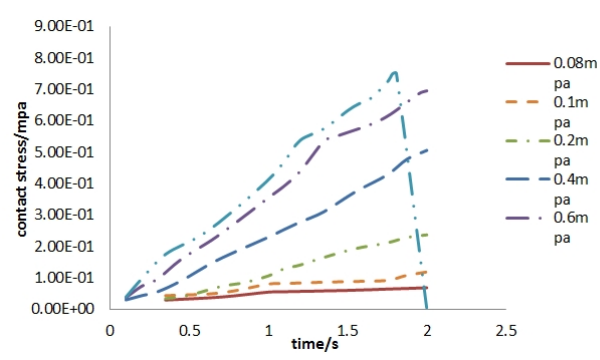

Fig.4 Contact stress in different inflation pressures

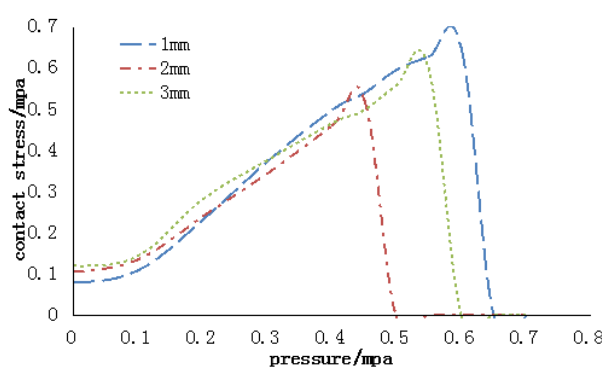

Fig.5 Contact stress under different assembly gap

Contact Stress And Shear Under Different Initial Compression Ratio. The rubber material under pressures may occur large deformation. If the shear stress exceeds the shear strength of rubber material, the ring would failure. Analyzing the maximum shear stress of the sealing ring under different compression ratios, and curves of the shear stress are obtained, as shown in Fig.6(b).

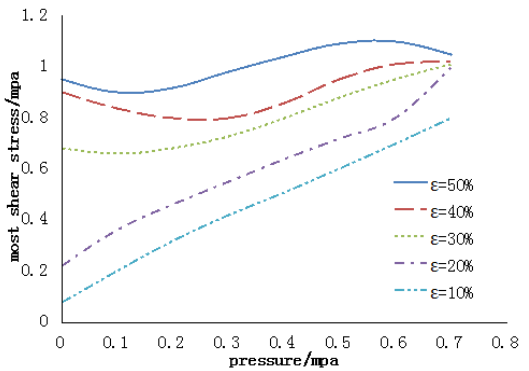

(a) Contact stress

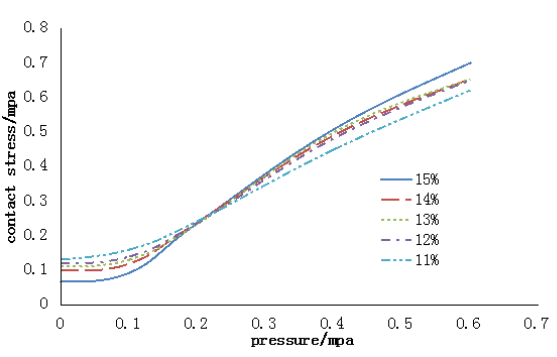

(b) Shear stress

Fig.6Stress under different compression ratios

By Fig. 6 (a), with the increasing of the compression ratio, the maximum contact stress changes more slowly. By Fig. 6(b), the maximum shear stress of the sealing ring is $1.12 \mathrm{mpa}$ when the inflation pressure under $0.7 \mathrm{MPa}$, the compression ratio under $15 \%$. Lessing than the shear strength 
$2.900 \mathrm{mpa}$. Sealing ring meets the strength requirements. The shear stress increases as the increasing of the compression ratio when inflation is constant. The compression ratio under $12 \%$, the shear stress increases obviously. When the compression rate is greater than $12 \%$, the shear stress increases less. When the compression ratio is greater than $14 \%$, the maximum shear stress tends to be stable. The initial compression rate is more than $12 \%$ usually. Excessive initial compression will cause the air bag to produce a large residual deformation, thus causing leakage. Compression rate should be chosen as small as possible in the premise of ensuring seal.

\section{Conclusions}

Inflation pressure, assembly gap and the initial compression ratio will affect the sealing effect of the T-ring.

We can know that the locations that most likely failure of the inflatable seal ring is also changing under different inflation pressures. The maximum stress initially focused on angles of the sealing ring, and gradually focused on the place where contacting with the sharp rigid members.

The inflation pressure too larger or too smaller may lead the seal ring failure. Optimum inflation pressure is $0.4 \sim 0.6 \mathrm{mpa}$ when draining pressure is $0.22 \mathrm{mpa}$ and shore hardness is HA 40 .

Under certain conditions, assembly clearance should design smaller within a reasonable range.

Within the normal range, the maximum contact stress is always greater than the operating pressure so that proper sealing properties can be ensured.

Inflation pressure is constant, the shear stress increases with the increasing of the compression rate. When the compression ratio is greater than $14 \%$, the shear stress increases significantly and tends to be stable. Considering all factors, compression ratio should choose small as far as possible under the premise of ensuring seal.

\section{References}

[1] Montgomery M E. Choke and kill lines-safety and downtime. Houston. IADC Deepwater Conference. Brookshire, Texas, August 27, 1998.

[2]Cary D N,Groomer V. Drilling riser alternative repair method for leaking joint seals utilizing pressure activatedsealant technology. IADC/SPE Drilling Technology Conference and Exhibition, paper 101216. Bangkok, Thailand, 2006.

[3] AI-Kharusi M S M, QamarS Z. Non-linear model for evaluation of elastomer seals subjected to differential pressure. SPE 149032, 2011.

[4] Meng Xiangkai, Wang Leqin. Nonlinear finite element analysis of inflatable seals. Lubricationand sealing, 32 (2007)110- 112.

[5] Ben Kecun, Tang Wencheng, Hong Xiangxu. Inflatable type flexible sealing of the nonlinear finite element analysis. Lubrication and sealing, 33 (3): 69 -71,2008.

[6] Zhou Zhihong, Zhang Kanglei, Li Jing, et al. The finite element analysis of the stress and contact stress of.0 shaped rubber sealing ring. Lubrication and sealing, 89 (4):86 ,2006.

[7] Deng Tao, Wang Wei, Zhao Shugao. Determination of material constants of rubber Mooney-Rivlin model. Special rubber products, 2004.

[8] Chen Guoming, Wen Jihong, Chang Yuanjiang. Riser joints $\mathrm{O}$ ring seal performance analysis. Petroleum machinery,41(1):45-58,2013. 